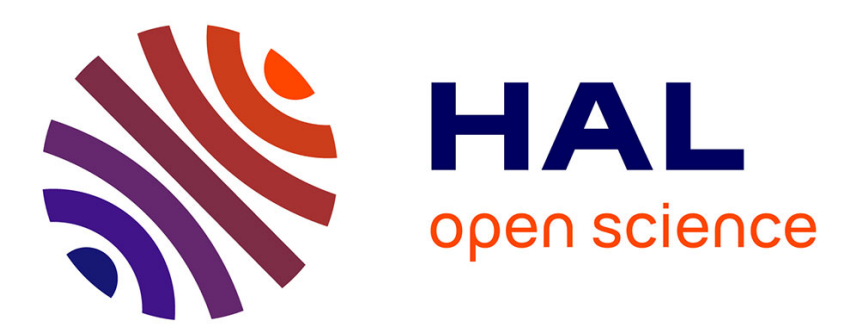

\title{
Experimental evidence of bifurcating nonlinear normal modes in piecewise linear systems
}

Oliviero Giannini, Paolo Casini, Fabrizio Vestroni

\section{To cite this version:}

Oliviero Giannini, Paolo Casini, Fabrizio Vestroni. Experimental evidence of bifurcating nonlinear normal modes in piecewise linear systems. Nonlinear Dynamics, 2010, 63 (4), pp.655-666. 10.1007/s11071-010-9827-y . hal-00630280

\section{HAL Id: hal-00630280 \\ https://hal.science/hal-00630280}

Submitted on 8 Oct 2011

HAL is a multi-disciplinary open access archive for the deposit and dissemination of scientific research documents, whether they are published or not. The documents may come from teaching and research institutions in France or abroad, or from public or private research centers.
L'archive ouverte pluridisciplinaire HAL, est destinée au dépôt et à la diffusion de documents scientifiques de niveau recherche, publiés ou non, émanant des établissements d'enseignement et de recherche français ou étrangers, des laboratoires publics ou privés. 


\title{
Experimental evidence of bifurcating Nonlinear Normal Modes in piecewise linear systems
}

\author{
Oliviero Giannini, Paolo Casini, Fabrizio Vestroni
}

\author{
Dipartimento di Ingegneria Strutturale e Geotecnica (DISG) \\ Università degli Studi di Roma "La Sapienza", \\ Via Eudossiana 18 - 00184 Roma, Italy \\ Email: p.casini@uniroma.it
}

Key words: Nonlinear Normal Modes, Piecewise linear systems, Damaged systems, Forced response, Experimental investigations.

\begin{abstract}
.
A system with piecewise linear restoring forces, typical of damaged beams with a breathing crack, exhibits bifurcations characterized by the onset of superabundant modes in internal resonance with significantly different shape than that of modes on fundamental branch.

A 2-DOF frame with piecewise linear stiffness is analyzed by means of an experimental investigation; the frame is forced by an harmonic base excitation and the operative modal shapes as well as the response amplitude are directly measured; the results are compared with numerical outcomes for different damping. This study shows that the shapes and the frequencies of certain Nonlinear Normal Modes (NNM) of the related autonomous system strongly affect the forced response both in the numerical and the experimental environment. Therefore, it is possible to match the NNM with the forced response of the system, leading to the prospective of identifying severity and position of the damage from experimental tests.
\end{abstract}

\section{Introduction}

The NNMs of a system [1,2], defined as synchronous periodic oscillation, are important because, in analogy to linear theory, resonance in forced systems typically occurs in the neighbourhood of NNM frequencies. Hence, knowledge of the normal modes of a nonlinear system can provide valuable insight regarding the position of its resonances, a feature of valuable engineering importance. Moreover, since the number of normal modes of a nonlinear system may exceed its degrees of freedom (superabundant NNMS), certain forced resonances are essentially nonlinear and have no analogies in linear theory; in such cases a linearization of the system might not be possible, or might not provide all the possible resonances that can be experienced.

In recent years, this item revealed its importance also for piecewise-smooth mechanical systems, PSS, governed by sets of ordinary differential equations which are smooth in regions of phase space: smoothness is lost as trajectories cross the boundaries between adjacent regions (discontinuity boundaries). Much effort in science and engineering has focused on this kind of problems [3]; typical mechanical applications include: oscillators colliding with a deformable or rigid stop [4, 5], beams with breathing cracks [6-9], stick-slip mechanical systems with friction [10-12].

Particular attention has been devoted to the investigation of the nonlinear modal properties of nonsmooth systems. To this end, according to the evolution of the classical Rosenberg's definition, the following generalization has been considered for these systems:

NNM is any periodic motion of the undamped autonomous system in which all generalized coordinates vibrate without necessarily passing through the zeros simultaneously $[9,13,14]$. 
When dealing with experimental campaign performed on nonlinear systems $[12,15,16,17]$, one faces the problem that investigating a real structure involves the everpresence of damping, and the information about the dynamics can be suitably retrieved through the forced response of the system. For this reason, the object of the study is not anymore an autonomous system and, thus, the concept of NNM must be carefully adapted and several philosophical rather than practical questions arise.

In a recent work [18] a general 2-DOF piecewise-smooth mechanical system with two damage parameters was investigated. This system is able to model the dynamics of an asymmetrically cracked cantilever beam vibrating in bending and hence exhibiting a bilinear stiffness. A general explanation of the basic nonlinear dynamics of these systems, and in particular, the onset of superabundant modes at internal resonances was presented; moreover, it was shown that the specific features of each bifurcation scenario peculiarly depends on the kind of the related internal resonance.

From a different perspective, the concept of NNM could be used for the identification of the damage in a structure from the observation of the nonlinear response of the system once excited. The basic idea behind it is that the nonlinear response of a real system can be linked to a nonlinear model and thus permits the identification of numerical parameters that govern the severity of the nonlinearity. Moreover, such nonlinearity can be a sharp index of the presence and of the evolution of a damage in the structure.

This paper is a step in this direction and presents a thorough investigation on the evidence of the nonlinear modes in the forced response of a simple two-degrees-of-freedom system. Basically, among the nonlinear normal modes of a given undamped system, only a subset is robust enough to survive once damping is considered. Moreover, an even smaller subset can be directly excited, because the energy of the external force tends to flow on the more responding modes. Thus, on a real environment, it is important to investigate and discriminate between persistent NNMs that contribute significantly to the response of the system and ghost $N N M s$ that derive from the periodical solution of the corresponding autonomous system without affecting the forced responses.

The paper is divided into three sections. The first section synthetically reports the equations and the bifurcation scenarios of the system at hand; a thorough presentation of this system can be found in [18]. The second section deals with the numerical simulation of forced response of the damped model, this section is devoted on one hand to analysing the expected scenarios of the experiments, on the other to address the discrimination between persistent and ghost NNMs. Finally, the third section is devoted to the experimental investigations.

\section{System Model}

\subsection{Equations of Motion}

The investigated system, Fig. 1, consists of a 2-DOF oscillator: two masses, $m_{1}$ and $m_{2}$, are connected by two piecewise linear springs, Fig. 1a, of undamaged stiffness $k_{1}$ and $k_{2}$, and damaged stiffness $\left(1-\varepsilon_{1}\right) k_{1}$ and $\left(1-\varepsilon_{2}\right) k_{2}\left(0 \leq \varepsilon_{\mathrm{i}}<1\right)$ : the relevant restoring forces exhibit the bilinear behaviour shown in Fig. 1b. The system is subjected to an harmonic base excitation $a_{\mathrm{g}}(t)=A \sin (\Omega \mathrm{t})$ where $A$ is the amplitude of the acceleration of the basement, while $\Omega$ is its frequency.

By assuming the displacements $x_{1}$ and $x_{2}$ as Lagrangian coordinates, the stiffness of the nonlinear springs can be represented, Fig. 1b:

$$
k_{b i l, i}=k_{i}\left(1-H\left(\eta_{i}\right) \varepsilon_{i}\right), \quad H_{i}=: H\left(\eta_{i}\right)=\left\{\begin{array}{ll}
1 & \eta_{i} \geq 0 \\
0 & \eta_{i}<0
\end{array} \quad i=1,2\right.
$$

where $\varepsilon_{1}$ and $\varepsilon_{2}$ are the damage parameters, $H$ is the Heaviside function, $\eta_{1}=x_{1}$ and $\eta_{2}=x_{2}-x_{1}$. 
Therefore in the configuration plane $\left(x_{1}, x_{2}\right)$, Fig. 1c, four regions with different stiffness properties are delimited by the following two boundaries:

$$
\Sigma_{1}:=\left\{x \in R^{2}: \eta_{1}(x)=0\right\}, \Sigma_{2}:=\left\{x \in R^{2}: \eta_{2}(x)=0\right\}
$$

As shown in Fig. 1c, in regions 1 and 3 the system exhibits only one spring with reduced stiffness at a time, whereas in region 2 and 4 the springs are both damaged or both undamaged respectively. As demonstrated in [19] and previously underlined in [18], for piecewise linear system, when the nonlinearities are concentrated at the origin and hence the switching hyperplane passes through the origin, the NNM frequencies are independent of the energy level, unlike polynomial nonlinear systems [20,21].

With reference to Fig. 1 and denoting by $\mathrm{q}$ the vector of Lagrangian coordinates, the following equations of motion in time domain are found:

$$
\mathrm{M} \ddot{\mathrm{q}}+\mathrm{D} \dot{\mathrm{q}}+\left(\mathrm{K}_{0}-\varepsilon_{1} H_{1} \mathrm{~K}_{1}-\varepsilon_{2} H_{2} \mathrm{~K}_{2}\right) \mathrm{q}=\mathrm{p} \sin (\Omega t)
$$

where $\mathrm{M}$ is the mass matrix, $\mathrm{D}$ is the damping matrix under the assumption of proportional damping, $\mathrm{K}_{0}$ is the stiffness matrix of the undamaged system and $\mathrm{p}$ collects the forcing amplitudes:

$$
\begin{gathered}
\mathrm{M}=\left[\begin{array}{cc}
m_{1} & 0 \\
0 & m_{2}
\end{array}\right], \mathrm{K}_{0}=\left[\begin{array}{cc}
k_{1}+k_{2} & -k_{2} \\
-k_{2} & k_{2}
\end{array}\right], \mathrm{K}_{1}=\left[\begin{array}{ll}
k_{1} & 0 \\
0 & 0
\end{array}\right], \mathrm{K}_{2}=\left[\begin{array}{cc}
k_{2} & -k_{2} \\
-k_{2} & k_{2}
\end{array}\right] \\
\mathrm{D}=\left[\begin{array}{cc}
c_{1}+c_{2} & -c_{2} \\
-c_{2} & c_{2}
\end{array}\right], \mathrm{q}=\left\{\begin{array}{l}
x_{1} \\
x_{2}
\end{array}\right\}, \mathrm{p}=-\left\{\begin{array}{l}
m_{1} A \\
m_{2} A
\end{array}\right\}:=\left\{\begin{array}{l}
P_{1} \\
P_{2}
\end{array}\right\}
\end{gathered}
$$

The NNMs for the system in Eq. (3) are computed for $\mathrm{D}=0$ and $\mathrm{P}=0$. The investigation is performed by using a numerical approach based on the search for fixed points of the Poincaré maps: by exploiting the properties recalled in the following subsection, the maps are obtained by assuming the energy level equal to the unity and considering maps with surface of section $\eta_{1}=0$ and $\dot{x}_{2}>0$.

\subsection{Free motion: Basic properties}

A thorough analysis, concerning the bifurcation events experienced by the NNMs of the system in Eq. (3), can be found in [18]. Some fundamental properties are recalled in the following [18,19,22,23]: i) since the nonlinear responses are independent of the energy level, as said before, the trajectories of NNM with the same frequency and different energy levels form a set of homothetic curves: thus, by fixing a certain level of the total energy, the initial conditions can be expressed by only one independent parameter; ii) the trajectories of NNMs in the configuration space are open curves and generally do not pass through the origin; iii) for most nonlinear systems the number of NNMs is at least equal to the number of normal modes of the underlying linear system: we denote these as fundamental NNMs with fundamental frequencies $\omega_{1}$ and $\left.\omega_{2} ; i v\right)$ the nonlinear frequency ratio $r=\omega_{2} / \omega_{1}$ only depends on the mass and stiffness ratios as well as on damage parameters $\varepsilon_{i}$, being $r_{0}$ the frequency ratio of the undamaged system; $v$ ) as the level of nonlinearity increases, additional NNMs (superabundant) generated by bifurcation mechanisms can be observed.

\subsection{Free motion: Bifurcating NNMs}

When $\varepsilon_{1}$ and/or $\varepsilon_{2}$ vary, first and second fundamental NNM frequencies vary generating ( $\left.n: m\right)$ internal resonances: this occurs when the nonlinear frequencies $\omega_{1}$ and $\omega_{2}$ are nearly commensurate i.e.: $n \omega_{1} \cong m \omega_{2}$ ( $n, m$ integers) [24-26]. Each internal resonance produces a 
specific structural change in Poincaré maps generally leading to new superabundant modes [18]. In particular it can be observed that, due to the different frequency ratio-damage laws, systems characterized by specified values of $r_{0}$ exhibit some internal resonances that are not possible for systems with different $r_{0}$ and vice-versa. In order to capture the expected scenarios of the experiments and without loss of generality, the numerical analysis is here performed in the case in which only one discontinuity boundary occurs: in more details, for damage parameters it will be assumed: $\varepsilon_{1}=\varepsilon \neq 0, \varepsilon_{2}=0$. Moreover, as far as the modal parameters are concerned, the following two values for the undamaged frequency ratio are considered: $r_{0}=2.62$, corresponding to a shear-type frame with $m_{1}=m_{2}$ and $k_{1}=k_{2}$, and $r_{0}=1.95$, below the 2:1 internal resonance. The following series of internal resonances will be considered: $(n: 1),(n: 2),(n: m)$ with $m>2$.

The $(n: 1)$ internal resonances with $n>2$ are first considered: this kind of resonances causes the occurrence of superabundant modes bifurcating from the backbone of the first mode. Correspondently the first NNM changes its shape remaining stable. Figures 2 and 3 refer to bifurcations occurring in $(n: 1)$ internal resonance $(n>2)$ for a system with $r_{0}=2.62$; in a system with different $r_{0}$ these could be qualitatively similar but, generally, take place for different values of $\varepsilon$. Figure 2 reports the frequency-damage plot with the various branches of $(n: 1)$ periodic solutions: there is a sequence of higher periodic NNMs bifurcating from the backbone of mode 1, called tongues. Each tongue takes place in the neighborhood of a $(n: 1)$ internal resonance and determines a qualitative change in the trajectory of the first NNMs: this can be deduced by the lower part of Fig. 2 where the evolution of the modal shapes (thick lines) are depicted together with the maximum equipotential boundaries (thin closed lines). It is worth noticing that, along the backbone of the first mode, a jump after each tongue arises generating an increasing number of inflections points in the modal curves [18]. Figure 3 describes in more detail the particular case (3:1): the frequency of the first fundamental NNM is plotted against the parameter $\varepsilon$, together with the modal shape. At $\varepsilon_{3: 1}$ stable and unstable superabundant NNMs $(\mathbf{C})$ with frequency $\omega_{1}$ generated by cyclic-fold bifurcations appear, whereas for increasing damage the nonlinear normal modes $\mathbf{A}$ become similar in shape to the second mode (B) and coalesces on it. The case of internal resonance condition with $n=2$ becomes possible for $r_{0}=1.95$; a characteristic example is presented in Fig. 4 . where the second mode experiences a period doubling bifurcation becoming unstable. For the case $\mathrm{r}_{0}=1.95$ the instability of the second NNM has been observed for a wide range of damage parameters: on the other hand, the second mode has been always found stable for systems with $\mathrm{r}_{0}>2$.

The $(n: 2)$ internal resonances affect the first mode that loses its stability and experiences a period doubling bifurcation; furthermore a second NNM appears and approaches the unstable NNM as long as the first mode recovers the stability. It should be highlighted that, unlike the previous cases, this bifurcation event is observable in a very narrow window of the damage parameter. As an example the case (7:2) can be found in [18].

Qualitatively different changes in the Poincaré maps are finally produced by $(n: m)$ resonances, with $m>2$. In these cases both fundamental modes remain stable whereas two pairs of stable and unstable superabundant NNMs appear and disappear; the frequency content in these modes are characterized by two main frequencies the ratio of which is exactly $n$-to- $m$. As an example the case (8:3) has been studied in [18].

\section{Forced Response}

\subsection{Persistent and Ghost NNMs}

By forcing a linear damped system with harmonic forces a steady state periodic response appears and, if the exciting frequency is close to a natural frequency of the system, in the 
configuration plane the forced response can resemble very closely the corresponding linear normal mode. When one deals with a nonlinear system, exciting a forced response that resembles a NNM is not trivial: first of all, the system can have more nonlinear natural frequencies than the degrees of freedom of the system and then can exhibit superabundant NNMs; moreover, some of these modes can be unstable and thus not excitable, but also, among the stable NNM some of them cannot be excited.

In the following, we will refer as Persistent Nonlinear Normal Mode (P-NNM) to the modes that can be retrieved in the forced motion of the system; moreover, these modes are the main cause of a peak in the system response. In Figure 5a a typical P-NNM is illustrated: Figures 5b-d report, for different damping, the trajectory of the steady-state responses obtained by forcing the system with driving frequencies equal to that of the fundamental NNMs. The other stable nonlinear normal modes, will be denoted as Ghost Nonlinear Normal Modes (G-NNM), to highlight that by observing the dynamics of the system from its forced response these modes do not appear and cannot be seen.

This section is devoted to the numerical investigation of the possibility of obtaining a harmonic forced response resembling the corresponding NNM in the configuration plane. For the sake of simplicity, four cases will be presented hereafter, the first three refer to the case of stable P-NNM while the latter presents the case of a stable G-NNM.

\subsection{Influence of the NNMs on the forced response}

A 2-DOF oscillator with different damping levels excited by an harmonic base excitation, $a_{\mathrm{g}}(t)=A \sin (\Omega t)$, is considered to investigate the counterpart of the phenomena illustrated in Subsection 2.3. As in the autonomous case, it will be assumed: $\varepsilon_{1}=\varepsilon \neq 0, \varepsilon_{2}=0, r_{0}=2.62$ or $r_{0}=1.95$ and a viscous proportional damping characterized by $c_{1}=c_{2}=c$.

The first example deals with the forced response for damage parameter near $\varepsilon_{3: 1}$, i.e. near a (3:1) internal resonance. Figure 6a is a colourmap in which the $x$-axis reports damage $\varepsilon$ whereas the $y$-axis refers to the excitation frequency $\Omega$ : each colour refers to the amplitude $x_{2}$ of the steady-state solutions starting from natural initial conditions. The solutions with higher amplitude, related to warmer colours, form branches which reveal the structure of the underlying autonomous system as become evident by comparing Fig. 6a to Fig. 3a. Furthermore the related curves in the configuration plane, Fig. 6b-g are qualitatively similar to the corresponding NNMs $\mathbf{A}$ and $\mathbf{C}$ of Fig. 3. In conclusion, since the forced responses exhibit the shapes of the NNMs $\mathbf{A}$ and $\mathbf{C}$, these are classified as P-NNM. The same case for different damping levels has also been examined; as expected for decreasing damping the branches become less fuzzy and their structure tends to the one of the corresponding autonomous system. Qualitatively similar remarks can be made for the responses around the other $(n: 1)$ internal resonances.

The forced response of the system, when the damage parameter is near the (2:1) internal resonance is then considered. As previously observed, this kind of resonance is possible, for example, in an oscillator characterized by $r_{0}=1.95$. Figures 7 presents the results of these simulations: Figure $7 \mathrm{a}$ depicts the colourmap, while the steady-state motions in the configuration plane relevant to the principal branches is presented in Figs. 7b-f. It can be noted that, at the value of damage causing the stability loss and period doubling of the second mode $\mathbf{B}$ in the autonomous system, Fig. 4, the forced solution of Fig. $7 \mathrm{~b}$ disappears and, for increasing damage, no longer appears. At the same time, two branches arise with motions both characterized by a period that is twice of that of the second mode and with shapes similar to the NNM $\mathbf{A}$ and $\mathbf{C}$ of Fig. 4. This behaviour persists even with large damping levels. Modes $\mathbf{A}$ and $\mathbf{C}$ are therefore persistent whereas mode $\mathbf{B}$ is a P-NNM provided its stability.

As a third example, Figure 8 refers to the dynamic scenario around a (7:2) internal resonance: according to the autonomous response, in a narrow window of damage, the first 
mode experiences a period doubling and then recovers its stability and its previous shape. In that narrow window, as long as the first NNM is unstable, the doubled period solutions of Figs. 8d and $8 \mathrm{f}$ are due to two superabundant NNMs of the corresponding autonomous system, which are persistent.

As a final remark, it is important to notice that any attempt to obtain forced response mimicking some other Nonlinear Normal Modes (e.g. in correspondence of the 8:3 internal resonance) failed. First of all, the system did not present any resonant behaviour; moreover, the system was responding with a deformed shape not resembling the corresponding autonomous NNM. Even in the case where the initial configuration of the system was set to match a modal deformed shape, after a reasonably small amount of cycles of the exciting force, the initial shape was lost and the system vibrated following the competing more robust NNMs. For this reason these modes are proposed to be referred to ghost modes, since it was not possible to see them through any periodical excitation of the system.

\section{Experimental set-up}

\subsection{Motivations}

As shown in the previous Sections, piecewise linear systems exhibit a large variety of dynamical behaviours, characterized by several occurrences of bifurcating nonlinear normal modes (NNMs).

The analysis of the undamped model provides scenarios with bifurcating NNMs that remain stable for a quite large range of the damage parameter. At the same time, the simulation of the damped model response to an harmonic force highlights that only some of these modes are indeed P-NNM and can be excited being dominant in the forced response, even in the case of a large system damping.

The experimental campaign aims at verifying experimentally the results obtained through the numerical integration of the equations of motion. In particular, it is of interest: $i$ ) the evaluation of the robustness of the nonlinear behaviour in a real environment, and ii) the possibility of evaluating the nonlinear mode through a measured forced response.

\subsection{Description}

The experimental rig, Fig. 9, consists of a two-storey shear-type frame (A) with two degrees of freedom $x_{1}$ and $x_{2}$. The first degree of freedom is connected to a beam (C) characterized by a piecewise linear stiffness. The shear type frame is constituted by two steel blocks $150 \mathrm{x} 50 \mathrm{x}$ $15 \mathrm{~mm}$ each characterized by a mass of $0.88 \mathrm{Kg}$, Fig. 10a.

Since the studied bifurcations occur at internal resonances, it is important to control in the experiments the initial ratio between the natural frequencies of the frame, i.e. the ratio $\omega_{2} / \omega_{1}$ when the system is undamaged. Therefore, the length of the supporting spring $l_{1}$ and $l_{2}$, as well as the extra mass that can be added to each steel block are chosen appropriately to have the desired initial ratio $r_{0}$, Figs. 10a,b.

The frame is attached to a vibrating bench that slides over two guides and is actuated by a shaker, Fig. 10c. Figure 11 shows a schematic drawing of the beam (C) characterized by a variable piecewise linear stiffness. The beam $\mathrm{C}$ is a cantilever beam, with the free edge connected to the first degree of freedom of the shear-type frame, Fig. $9 \mathrm{~b}$.

The beam is in contact, on one side, with a block that is connected to the bench, and, on the other side, to a sliding block (B), Fig. 11. The block B can slide along the axis of the beam; the stiffness of the beam is lower when positive displacements of the free edge occur. By adjusting the position of the block $\mathrm{B}$ one can vary the ratio between $k_{1}$ and $k_{1}{ }^{*}$ and thus control the severity of the nonlinearity of the system, i.e. the damage factor $\varepsilon$. Figure $12 \mathrm{a}$ 
shows how $k_{1}$ and $k_{1}{ }^{*}$ decrease as $l^{*}$ increases while Figure $12 \mathrm{~b}$ shows the corresponding change in damage factor $\varepsilon$.

The rig is equipped with two accelerometers (see Fig. 10a), one on each storey of the frame, that measure the vibrations of the two degrees of freedom. The accelerometers are connected to a charge amplifier that also provides two analogical integration of the acceleration signals so that both acceleration and displacement of the degrees of freedom are directly measured, avoiding the use of numerical integration of sampled signals. A third accelerometer is placed on the vibrating bench to provide a reference signal and a direct measure of the excitation amplitude (Fig. 10c).

The signals are sampled at $1 \mathrm{kHz}$, the digital recorder has parallel $\mathrm{A} / \mathrm{D}$ converters and apply the anti-aliasing filters on the analogue signals.

\subsection{Methodology}

In order to experimentally build the bifurcation diagrams, the vibrating bench is actuated with a slowly varying sine sweep (about $0.04 \mathrm{~Hz}$ per second) in order to assure that "almost stationary" conditions occurs. Each frequency sweep covers a range of about $5 \mathrm{~Hz}$ with a central frequency selected to follow the studied bifurcating natural frequency of the system.

After each measurement, the position of the block B is changed, and another sweep is performed for a different damage factor $\varepsilon$.

In analogy with the numerical outcomes, the experimental results are presented by means of colourmaps of the forced response for varying frequency and damage; these plots highlight that in the case of P-NNM the system response can be quite large, for both bifurcating modes. Moreover, the forced responses of the two degrees of freedom can be plotted on the configuration plane and compared with both theoretical and numerical results showing how a P-NNM can indeed be detected from experiments.

In order to be able to obtain the experimental bifurcation diagram, it is necessary that the target dynamical behaviour is stable and persistent for a reasonably large range of the damage parameter. For this reason, among the bifurcating scenarios presented by the studied dynamical system, experiments focus on two of them: the 3:1 and the 2:1 internal resonances.

\subsection{Experimental results: bifurcation 3:1}

In order to study experimentally the bifurcation $3: 1$, it is necessary to have an initial ratio $r_{0}=\omega_{1} / \omega_{2}$ smaller than 3 . In fact, by increasing the damage factor, both $\omega_{1}$ and $\omega_{2}$ decrease, but the latter one decreases more slowly than the former, causing the ratio $r$ to increase. In the experiments, the prototype has an initial ratio $r_{0}$ equal to 2.4.

Figure 13a shows the colourmap of the experimental forced response $x_{2}$ for an exciting frequency ranging $4 \mathrm{~Hz}$ centred in $\omega_{1}$. It is possible to easily follow the first natural frequency that decreases as the damage increases; once $\omega_{1}$ becomes close to $\omega_{2} / 3$ the peak in the forced response splits into two, clearly detecting the underlying bifurcation. By plotting the response of the two degrees of freedom, in the configuration plane, $x_{1}-x_{2}$, it is possible to follow the evolution of this P-NNM, Fig. 13b-g, and the occurrence of the superabundant mode.

Following the main branch, one can see the first mode shape gradually changing into the mode A of Fig. 3. At the same time a new periodic shape arises matching the P-NNM C. It is important to notice that, as expected from model and numerical simulation, for a quite large damage range, both modes $\mathbf{A}$ and $\mathbf{C}$ can be excited, causing, furthermore, a large response of the system for a wide frequency range.

\subsection{Experimental results: bifurcation 2:1}

The experimental evaluation of the bifurcation occurring in correspondence of the internal resonance 2:1, requires an initial ratio $r_{0}$ slightly below 2 . In the experiments, as in the 
numerical investigations, the ratio $r_{0}$ of the model is equal to 1.95. In this case, the measurements were aimed at detecting the period doubling of the second mode, thus the analysed frequency range is set around the second natural frequency of the system at about $43 \mathrm{~Hz}$.

The expected behaviour of this system for increasing damage along the fundamental branch is that suddenly the second mode should become unstable around the $2: 1$ internal resonance condition, while two stable P-NNM arise both characterized by a period that is twice the second mode period.

Figure 14a presents the colourmap of the experimental forced response. By examining the responses of the two degrees of freedom in the configuration plane, Figs 14b-d, it is possible to detect the destabilization of the second mode that is the only responding mode in this frequency range when damage is below 0.3: it suddenly becomes unstable for larger damage values and two 2T stable NNMs are detected, according to the numerical outcomes in Fig. 7. As for the previous case, the P-NNM are characterized by a large response of the system and are thus easily detectable from the harmonic response.

\section{Conclusions}

The experimental investigation carried out on a 2-DOF piecewise-linear system shows a strong nonlinear behaviour; such nonlinear behaviour can be relevant even for a small value of the damage parameter and increases with it. Moreover, the frame model can be excited so that the response of the system exhibits in the configuration plane a shape that mimics the mode shape of the related autonomous system. With reference to the development of damage detection strategies based on the identification on NNM, it is necessary, in the first place, to know which modes can be excited and which cannot, since only excitable modes can be measured and thus identified.

The experimental investigations confirm that the NNMs can be classified among persistent and ghost modes, being possible to excite the formers while the latter ones do not appear in the forced response of the system. Moreover, in order to experimentally evidence a bifurcation phenomenon and evaluate the persistence of a given mode, it is also necessary that such a mode remains persistent for a quite large range of values of the damage parameter; thus, numerical simulations are carried out and presented to improve the readability of the measurements. The experimental results generally agree with the numerical simulations, so that the following statements can be made as results of the presented study:

1. The fundamental modes are always persistent, provided their stability.

2. The stable superabundant modes around the $n: 1$ internal resonances are observed to be always persistent and excitable at the natural frequency of the first nonlinear mode. The 2:1 bifurcation presents a particular behaviour: since the second mode becomes unstable, the superabundant mode is persistent and excitable at the natural frequency of both the first and the second modes due to the period-doubling phenomenon.

3. As observed in numerical simulations and confirmed by the experiments, the superabundant modes are always persistent when the fundamental mode, experiencing a bifurcation event, becomes unstable; this occurs as an example around the $n: 2$ internal resonances.

4. Around the $n: m$ internal resonances $(n>2, m>2)$, the superabundant modes are ghost (G-NNM) whenever the fundamental modes remain stable.

The previously observed phenomena could provide additional information to explain the experimental measurements of real damaged structures leading to the prospective of identifying severity and position of the damage from the observation of the nonlinear response of the system to external excitations. 


\section{ACKNOWLEDGEMENTS}

This research was partially supported by the FY-2007/08 grant PRIN 2007.

\section{REFERENCES}

[1] R.M. Rosenberg: On normal vibrations of a general class of nonlinear dual-mode systems. Journal of Applied Mechanics, 29, 7-14 (1962)

[2] A.F. Vakakis: Non-linear normal modes and their applications in vibration theory: an overview. Mech. Syst. Signal. Process. 11, 3-22 (1997)

[3] R. Leine and H. Nijmeijer: Dynamics and bifurcations of non-smooth mechanical systems. Springer (2007)

[4] U. Andreaus and P. Casini: Dynamics of SDOF oscillators with hysteretic motion limiting stop. Nonlinear Dynamics. 22, 155-174 (2000)

[5] S. Natsiavas: Dynamics of Multiple Degree of Freedom Oscillators with Colliding Components. J. Sound Vib. 165, 439-453 (1993)

[6] Y.C Chu and H.H. Shen: Analysis of forced bilinear oscillators and the application to cracked beam dynamics. AIAA Journal 10, 2512-2519 (1992)

[7] M. Chati, R. Rand, and S. Mukherjee: Modal analysis of a cracked beam. J. Sound Vib., 207(2), 249-270 (1997)

[8] U. Andreaus, P. Casini, F. Vestroni: Non-linear dynamics of a cracked cantilever beam under harmonic excitation. Int. J. Non-Linear Mech. 42(3), 566-575 (2007)

[9] F. Vestroni, A. Luongo, and A. Paolone: A perturbation method for evaluating nonlinear normal modes of a piecewise linear 2-DOF system. Nonlinear Dynamics, 54, 379-393 (2008)

[10] P. Casini and F. Vestroni: Nonstandard bifurcations in mechanical systems with multiple discontinuity boundaries. Nonlinear Dynamics 35, 41-54 (2004)

[11] F. Svahn and H. Dankowicz: Energy Transfer in Vibratory Systems with Friction due to Lowvelocity Collisions. Journal of Vibration and Control, 14, 255-284 (2008).

[12] P. Casini, O. Giannini, F. Vestroni: Experimental evidence of non-standard bifurcations in non-smooth oscillator dynamics, Nonlinear Dynamics (2006) 46:259-272

[13] G. Kerschen, M. Peeters, J.C. Golinval, A.F. Vakakis: Nonlinear normal modes, Part I: A useful framework for the structural dynamicist. Mechanical Systems and Signal Processing, 23( 1), 170-194 (2009)

[13] M. Peeters, R. Viguie, G. Serandour, G. Kerschen, J.-C. Golinval: Nonlinear normal modes, Part II: Toward a practical computation using numerical continuation techniques. Mechanical Systems and Signal Processing, 23(1), 195-216 (2009)

[15] E. Gourdon, N.A. Alexander, C.A. Taylor, C.H. Lamarque, S. Pernot: Nonlinear energy pumping under transient forcing with strongly nonlinear coupling: Theoretical and experimental results. J. Sound Vib. 300(3-5), 522-551 (2007)

[16] R.H.B. Fey, R.M.T. Wouters, H. Nijmeijer: Proportional and derivative control for steadystate vibration mitigation in a piecewise linear beam system. Nonlinear Dynamics. DOI 10.1007/s11071-009-9613-x (2009)

[17] X. Jiang, D.M. McFarland, L.A. Bergman, A.F. Vakakis: Steady state passive nonlinear energy pumping in coupled oscillators: Theoretical and experimental results. Nonlinear Dynamics, 33 (1), 87-102 (2003)

[18] P. Casini and F. Vestroni: Characterization of bifurcating Nonlinear Normal Modes in piecewise linear mechanical systems. Int. J. Non-Linear Mech. (Submitted)

[19] L. Zuo and A. Currier: Non-linear and complex modes of conewise linear systems. J. Sound Vib. 174, 289-313 (1994)

[20] O. Gendelman, L.I. Manevitch, A.F. Vakakis, L.A. Bergman: A degenerate bifurcation structure in the dynamics of coupled oscillators with essential stiffness nonlinearities. Nonlinear Dynamics, 33 (1), 1-10 (2003)

[21] Y.S. Lee, G. Kerschen, A.F. Vakakis, P.N. Panagopoulos, L.A. Bergman, D.M. McFarland: Complicated dynamics of a linear oscillator with a light, essentially nonlinear attachment. Physica D, 204 (1-2), 41-69 (2005) 
[22] S. Chen and S.W. Shaw: Normal modes for piecewise linear vibratory systems. Nonlinear Dynamics 10, 135-163 (1996)

[23] E.A. Butcher and R. Lu: Order reduction of structural dynamic systems with static piecewise linear nonlinearities. Nonlinear Dynamics, 49, 375-399 (2007).

[24] A.H. Nayfeh, C. Chin, S.A. Nayfeh: On nonlinear normal modes of systems with internal resonance. J. Vib. Acoust. 118, 340-345 (1996)

[25] D. Jiang, C. Pierre, S.W. Shaw: The construction of non-linear normal modes for systems in internal resonance. Int. J. Non-Linear Mech., 40, 729-746 (2006).

[26] C.H. Pak: On the coupling of non-linear normal modes, Int. J. Non-Linear Mech., 41, 716725 (2006) 


\section{FIGURE CAPTIONS}

Figure 1. a) System model; b) piecewise restoring forces $\left(\eta_{1}=x_{1} ; \eta_{2}=x_{2}-x_{1}\right)$; c) discontinuity boundaries in the physical plane

Figure 2. Evolution of the first fundamental frequency $\left(r_{0}=2.62\right)$ for varying $\varepsilon_{1}=\varepsilon$ and relevant modal curves

Figure 3. System $r_{0}=2.62$ : a) frequency- $\varepsilon$ branching diagram (solid lines refer to stable NNMs); b) relevant modal curves around the 3:1 internal resonance

Figure 4. System $r_{0}=1.95:$ modal curves and Poincaré sections around the 2:1 internal resonance

Figure 5. System $r_{0}=2.62, \varepsilon_{2}=0$ : a) P-NNM for $\varepsilon_{1}=0.55$; b)-d) steady state responses to harmonic excitation for different damping parameters $c_{1}=c_{2}=c$

Figure 6. Numerical forced response around the 3:1 internal resonance, $r_{0}=2.62:$ a) Coloured map; b)-g) steady state responses in the configuration parameters

Figure 7. Numerical forced response around the 2:1 internal resonance, $r_{0}=1.95:$ a) Coloured map; b)-g) steady state responses in the configuration parameters

Figure 8. Forced response around the 7:2 internal resonance, $r_{0}=2.62$ : a) Coloured map; b)-g) steady state responses in the configuration parameters

Figure 9. Experimental set-up: a) global view; b) outline

Figure 10. Experimental get-up. Details

Figure 11. Schematic view of the bilinear stiffness beam

Figure 12. a) Bilinear stiffness of the beam for varying $l^{*}\left(k_{1}\right.$ for $x_{1}>0, k_{2}$ for $\left.x_{1}<0\right)$. b) Damage as a function of $l^{*}$

Figure 13. Detail of the bifurcation for the internal resonance at $\omega_{1}=\omega_{2} / 3$ with the corresponding modal shapes

Figure 14. Detail of the bifurcation for the internal resonance at $\omega_{1}=\omega_{2} / 2$ with the corresponding modal shapes 


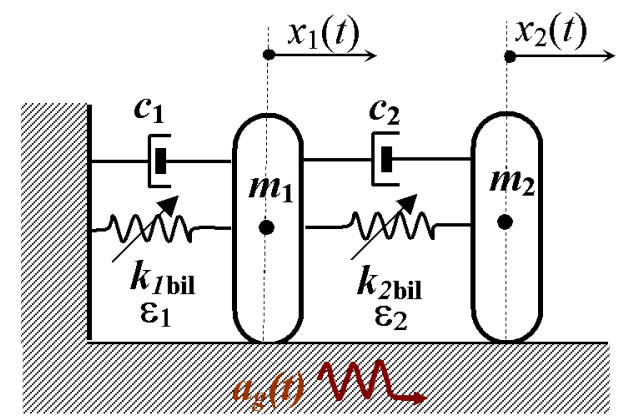

a)

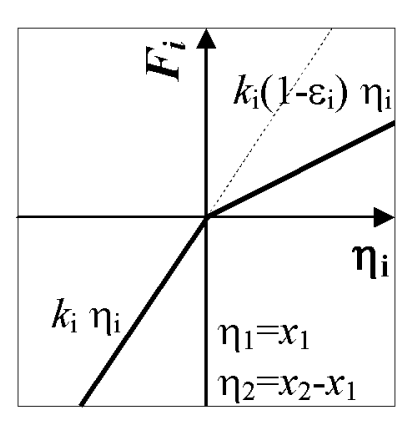

b)

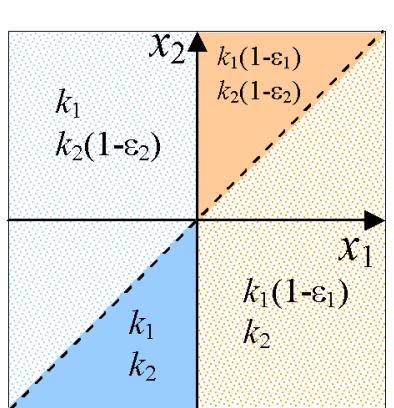

c) 

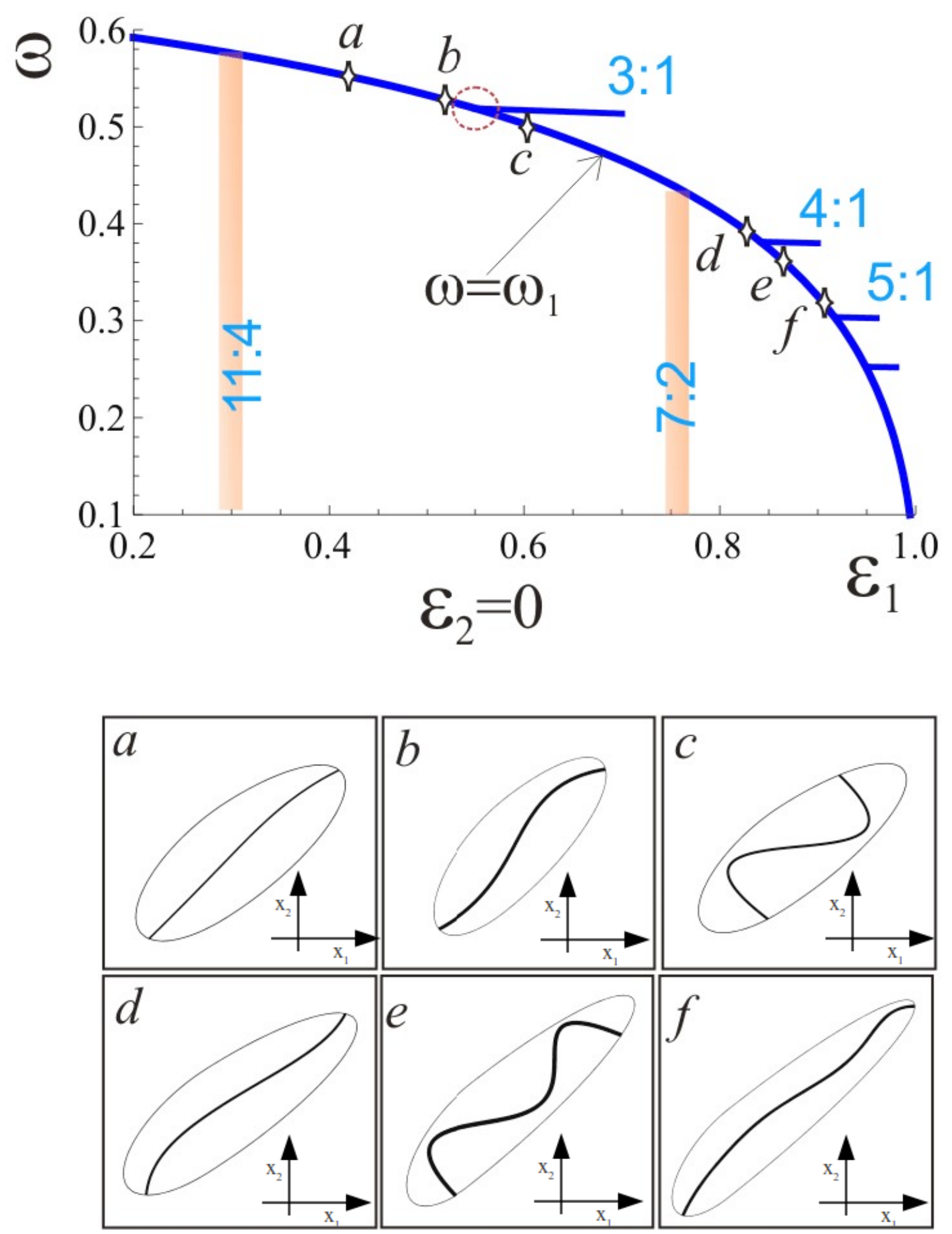

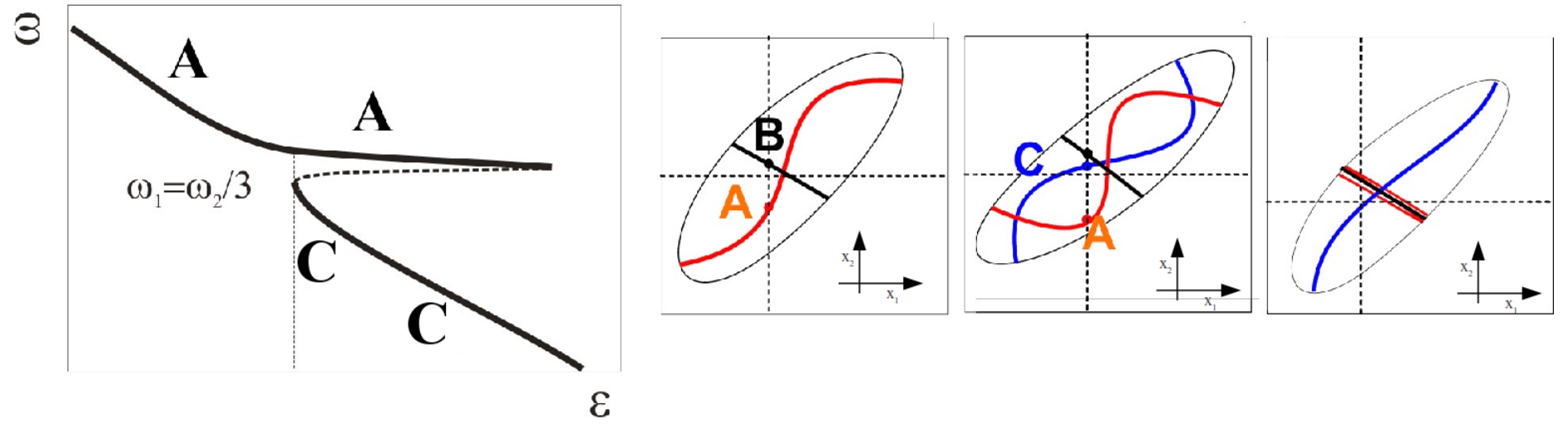

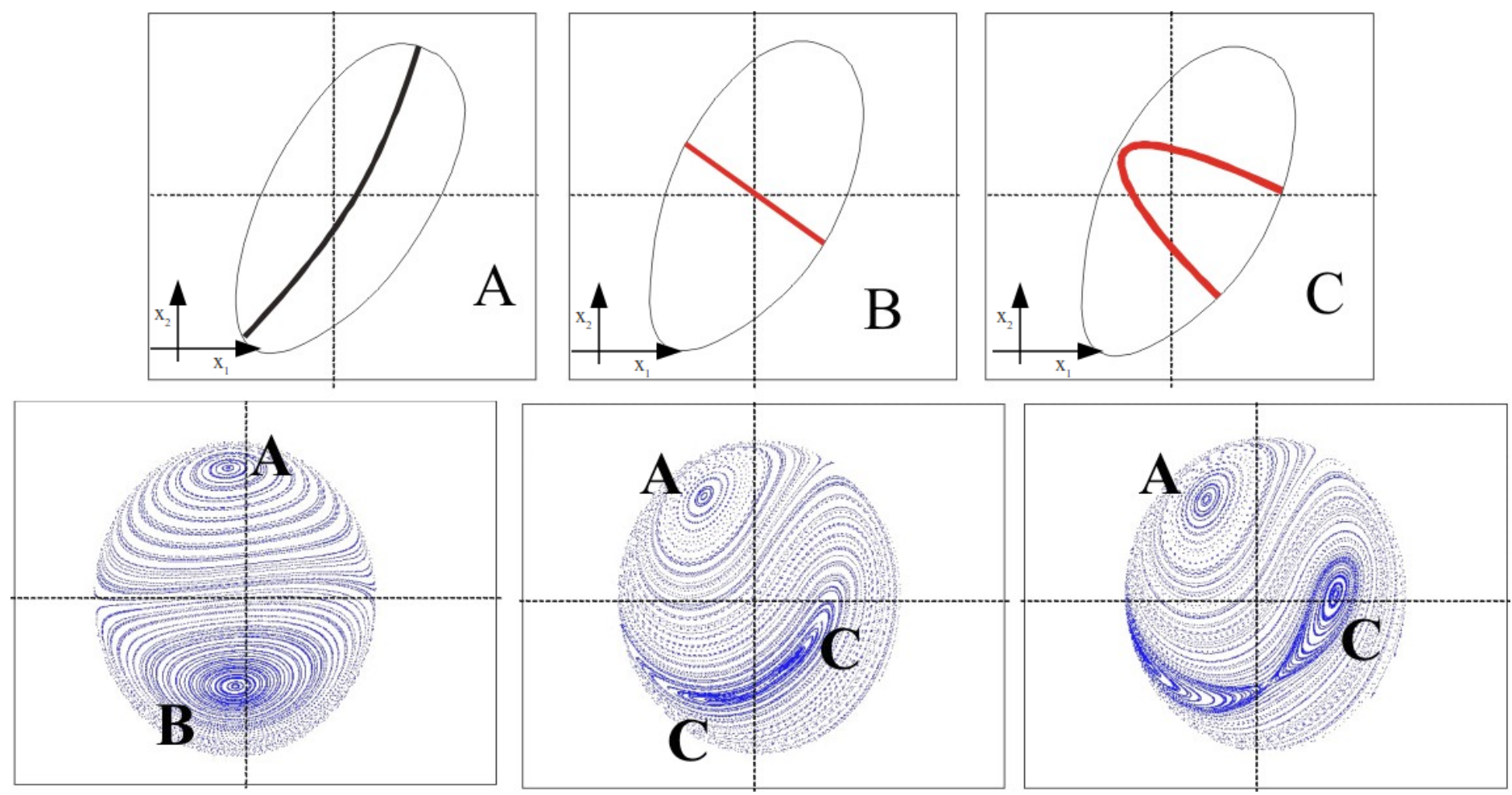


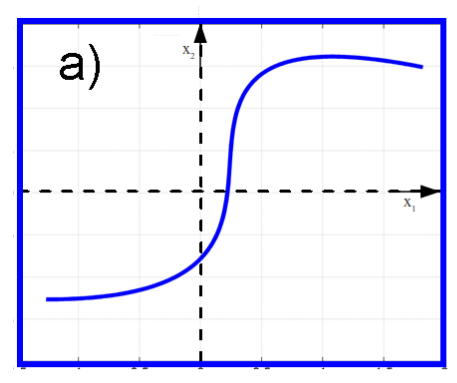

P-NNM

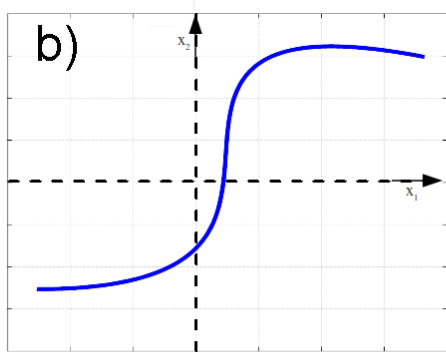

$c=0.001 \mathrm{Nsm}^{-1}$

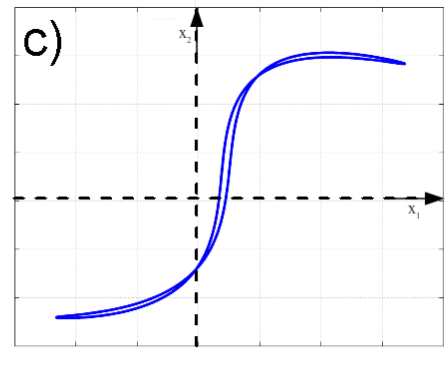

$c=0.005 \mathrm{Nsm}^{-1}$

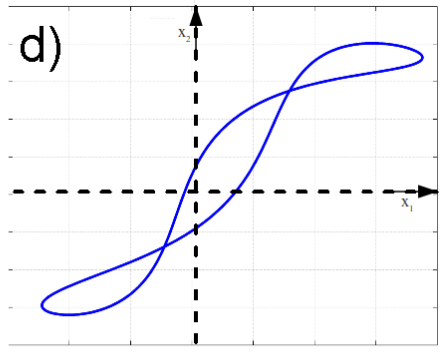

$c=0.05 \mathrm{Nsm}^{-1}$ 

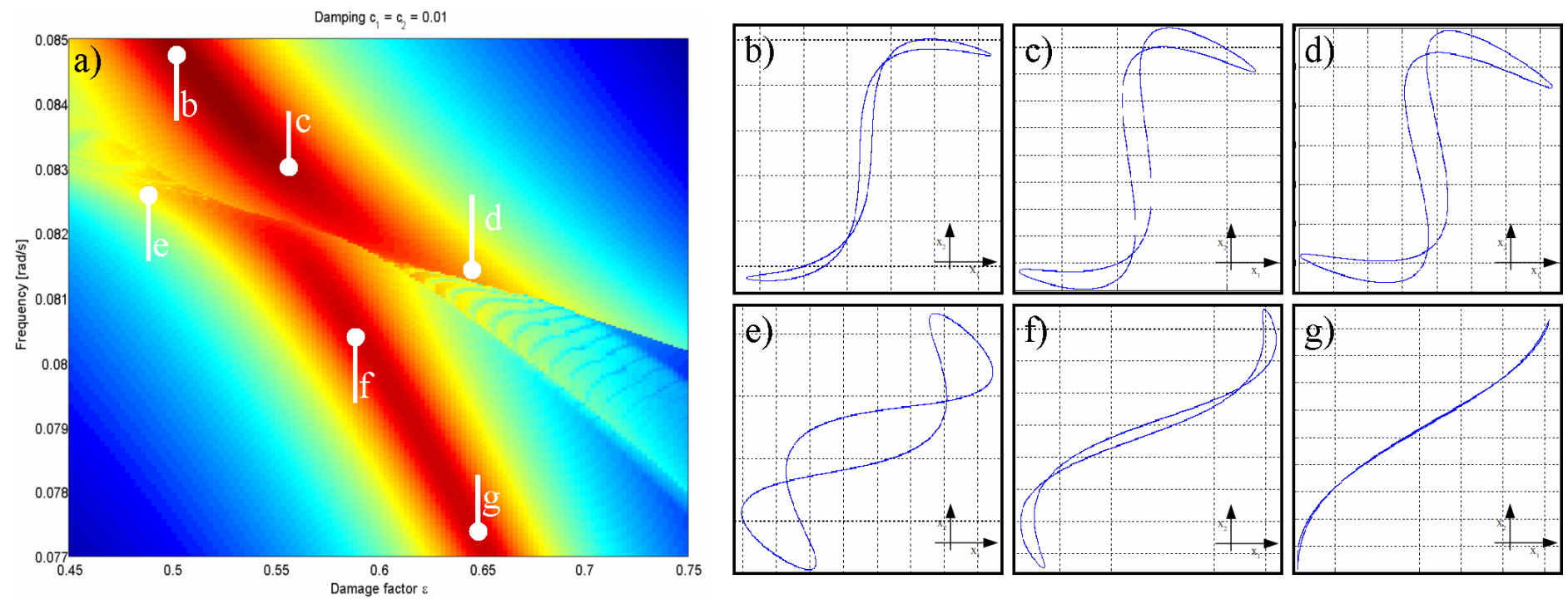


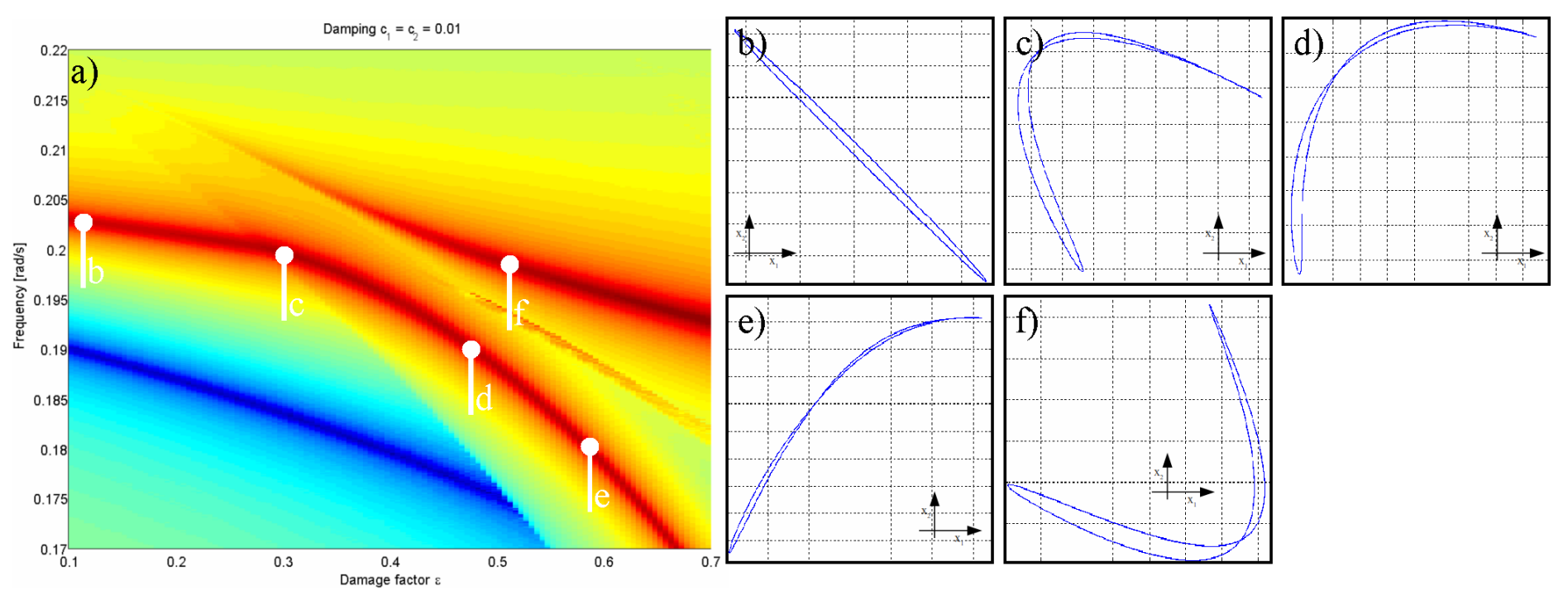



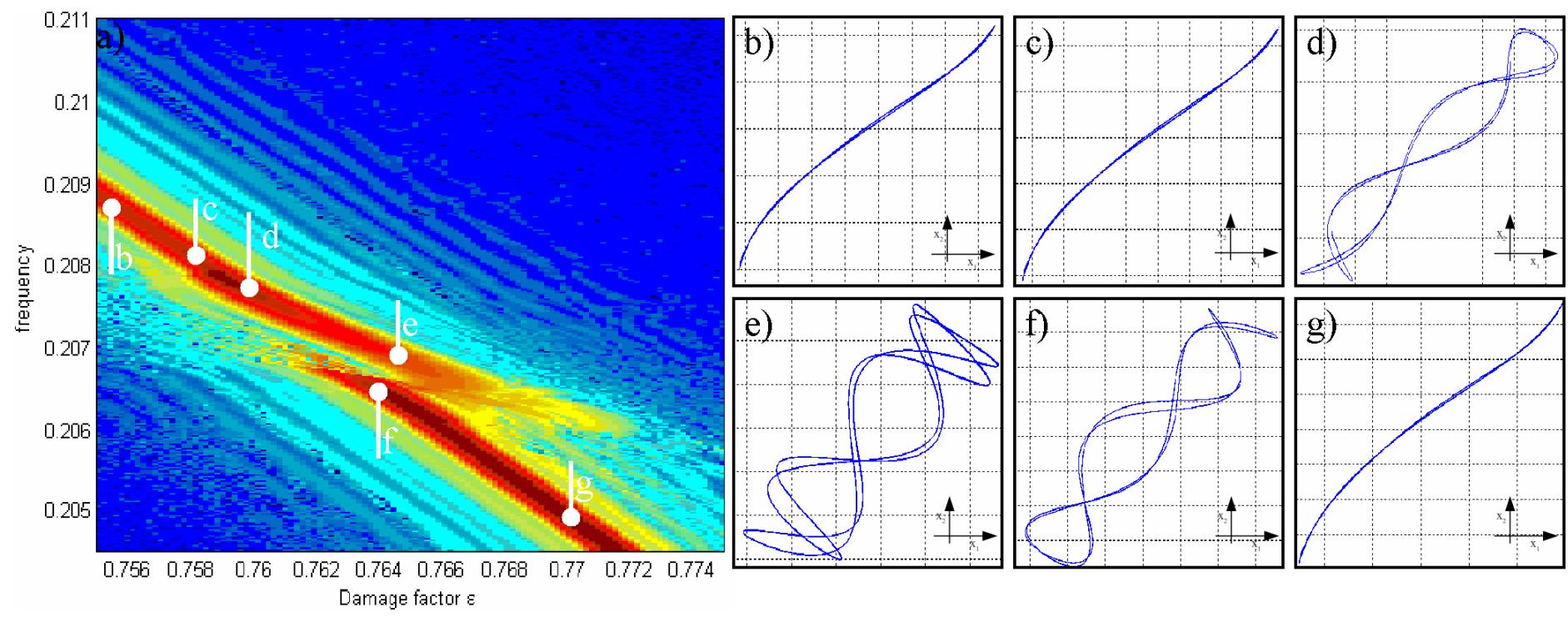


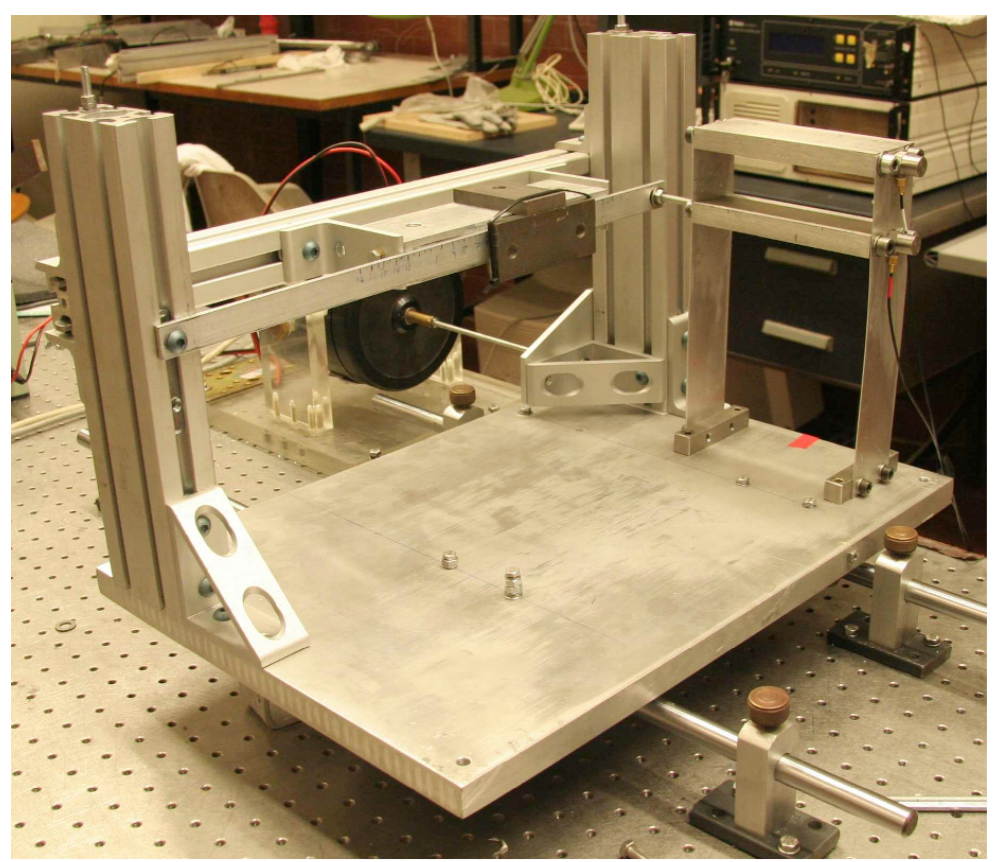

a)

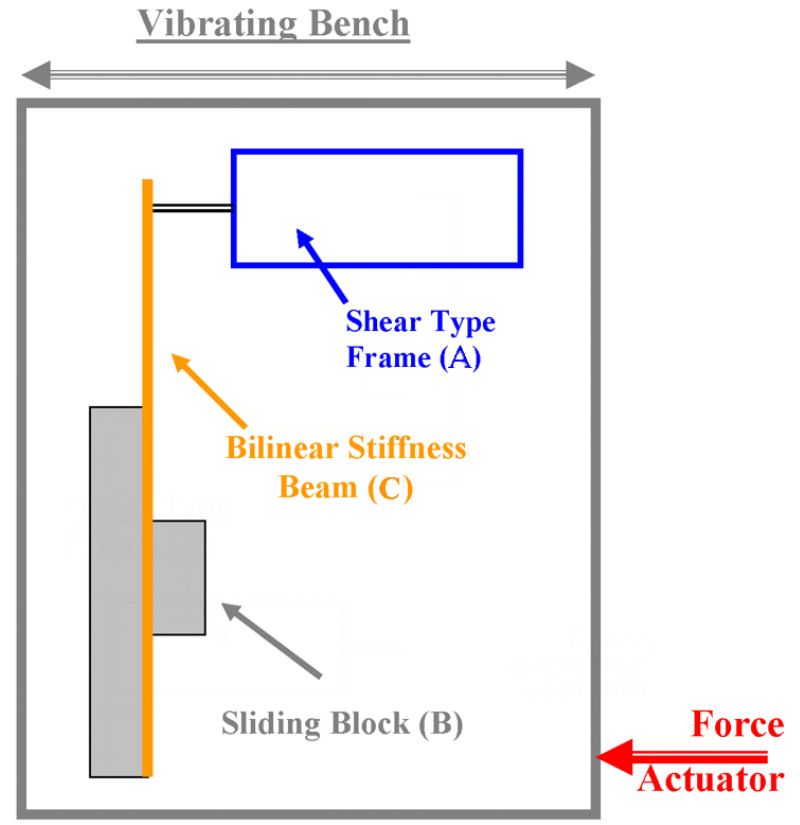

b) 

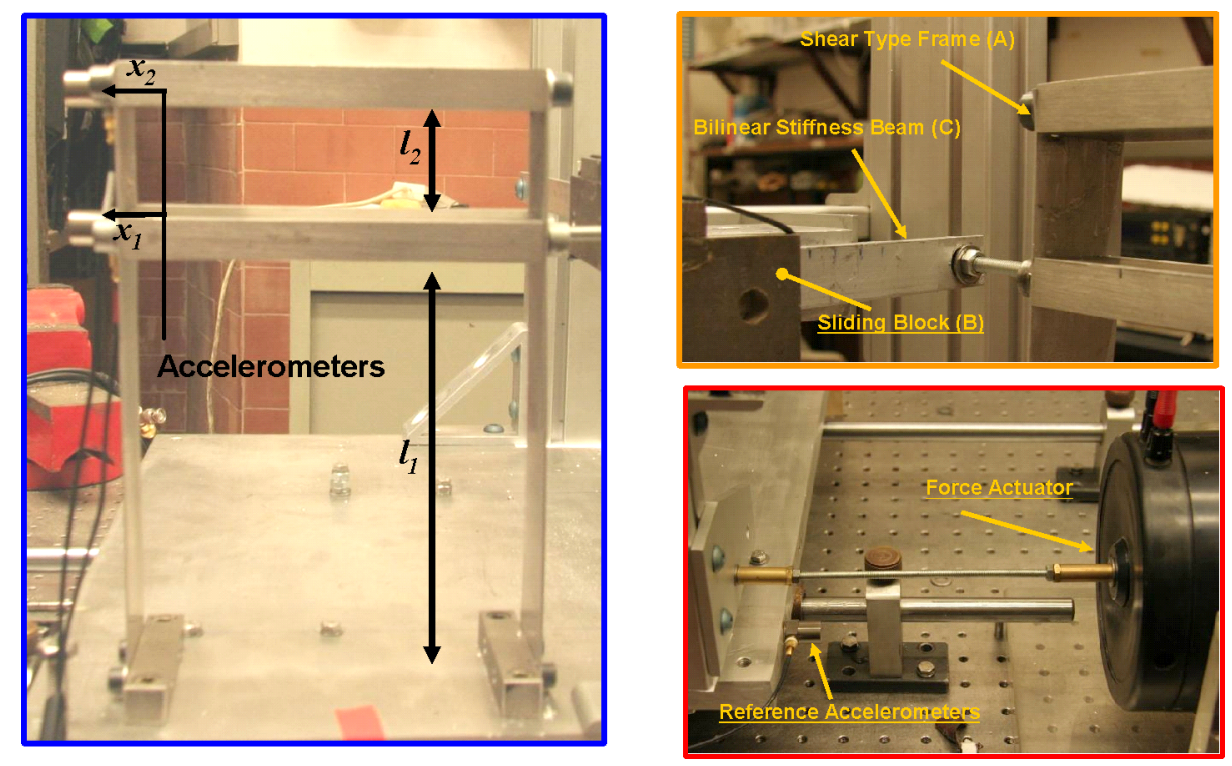


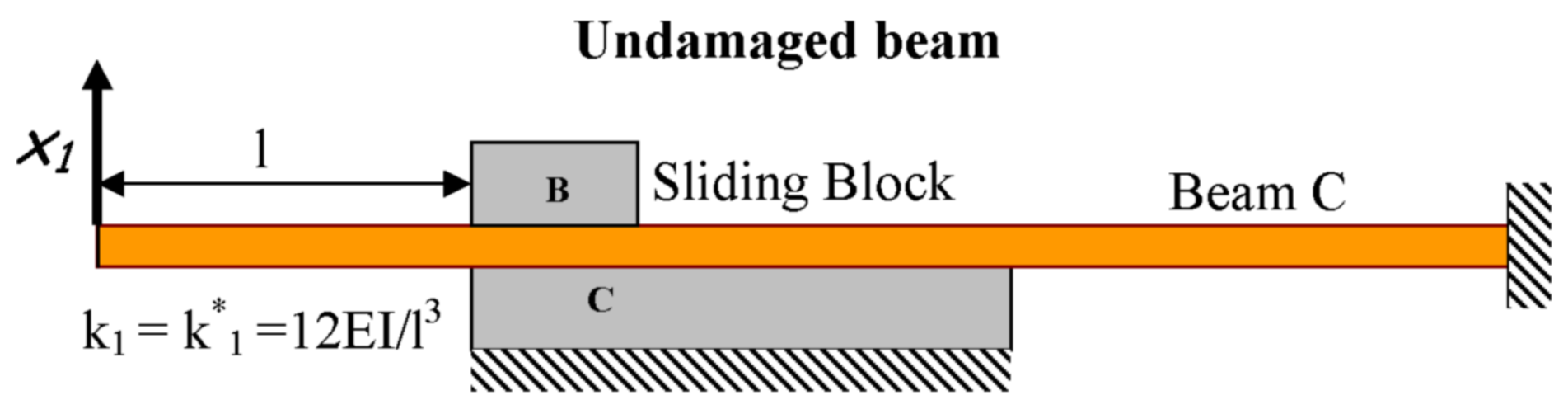

\section{Damaged beam}

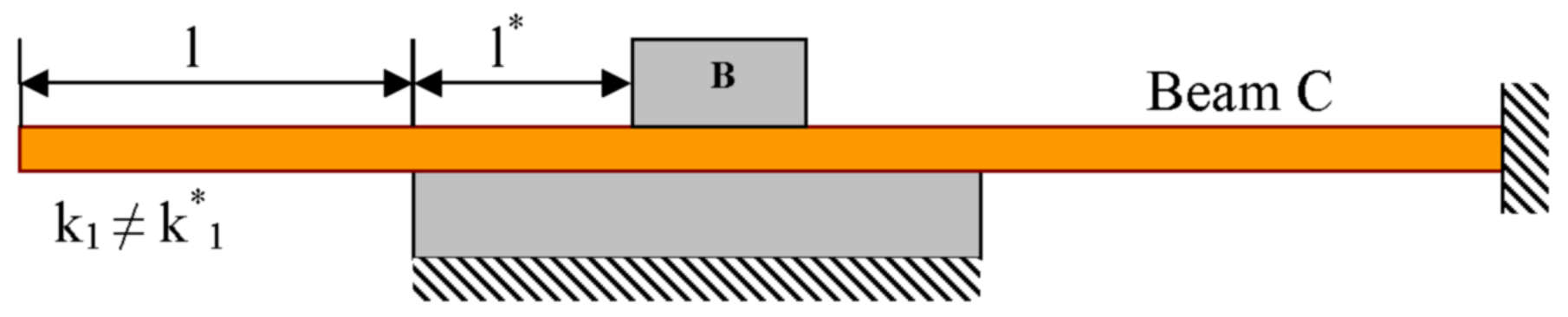



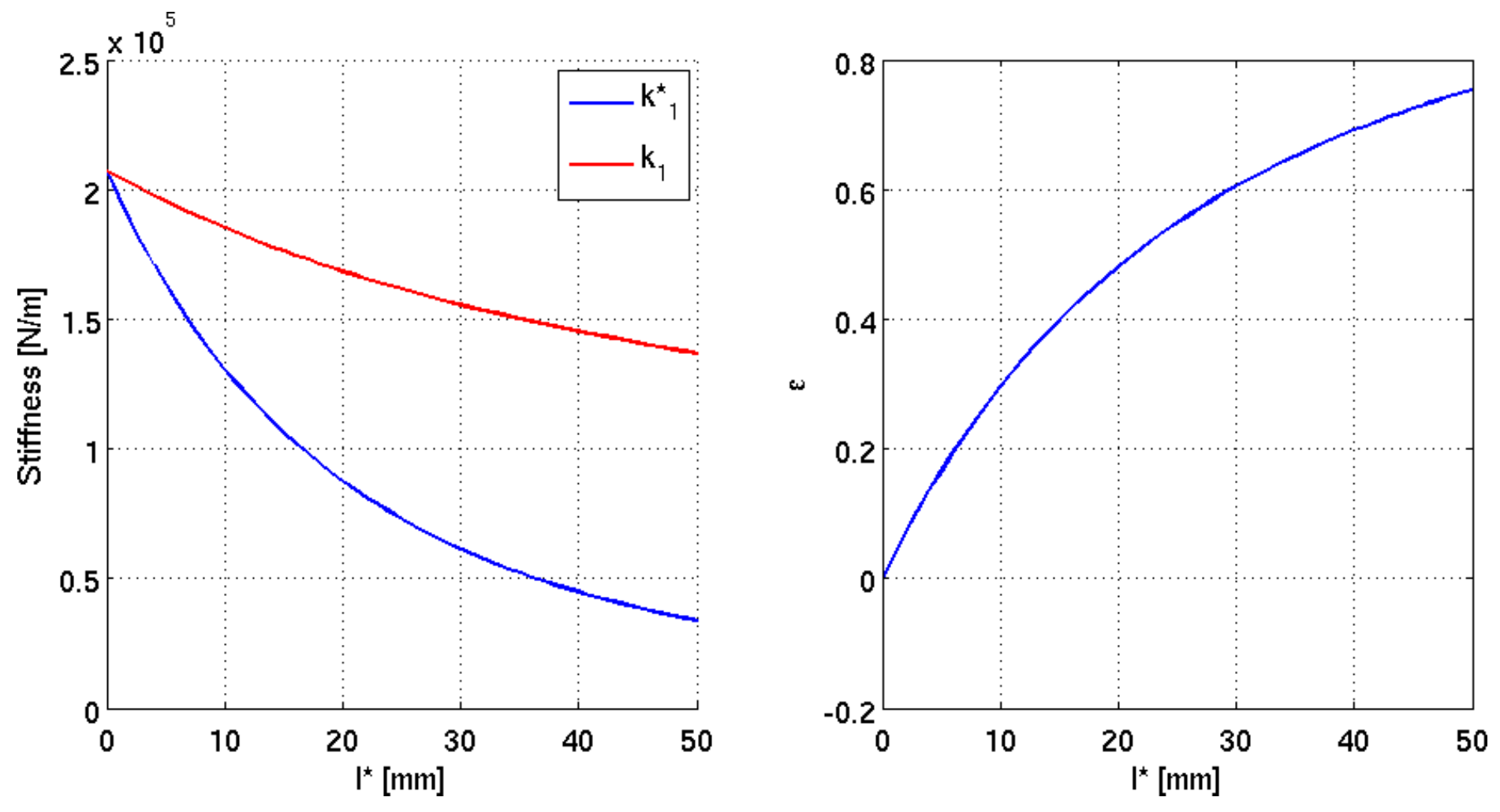

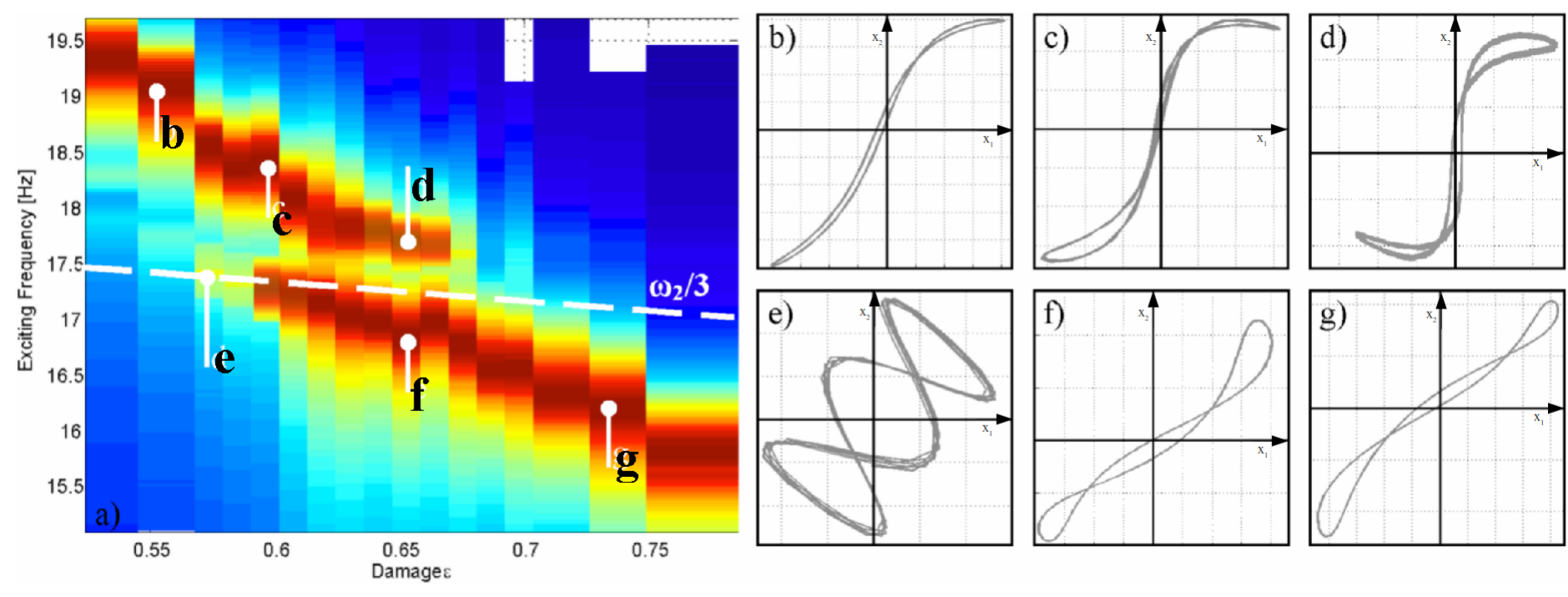

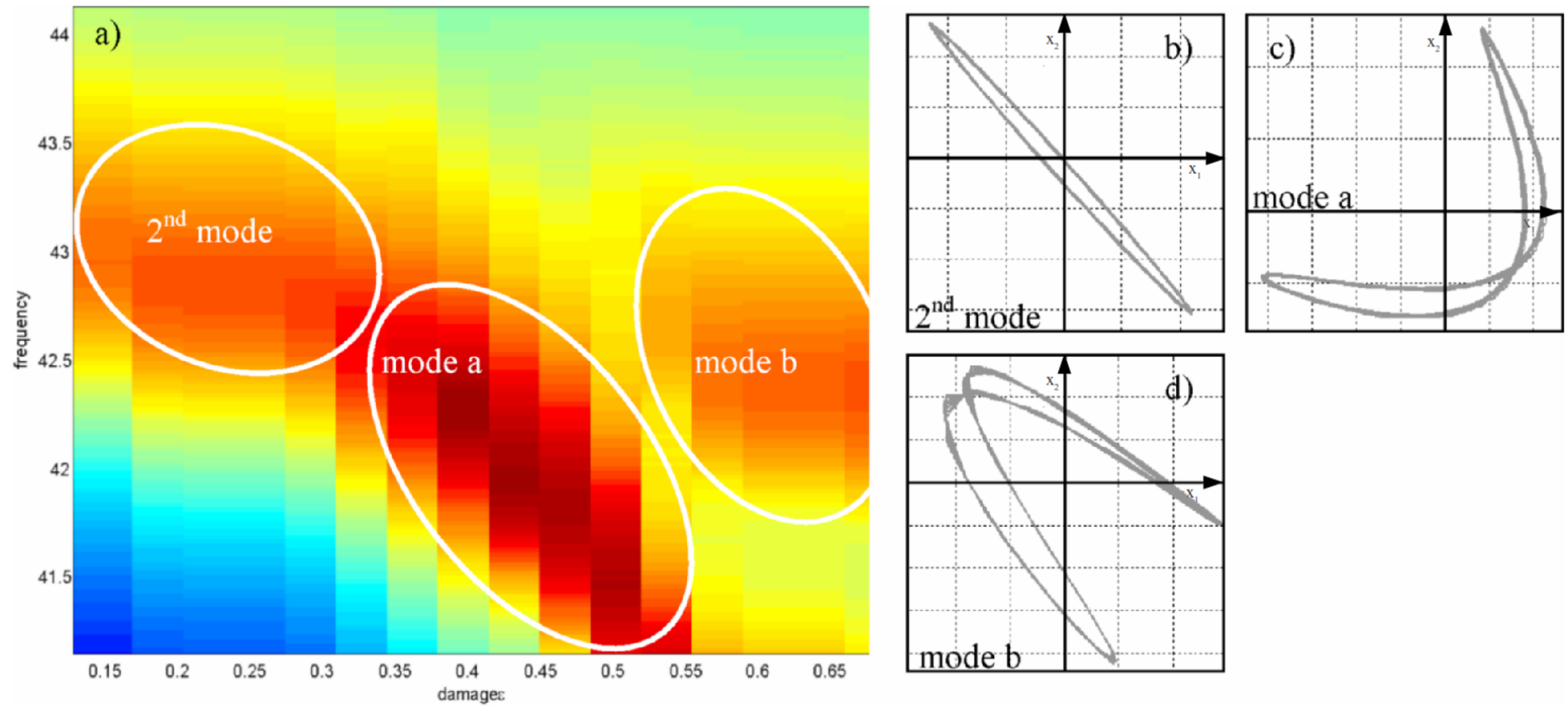